\title{
ANUNCIO DE FUSIONES Y ADQUISICIONES Y SU EFECTO EN LOS RETORNOS ACCIONARIOS: CHILE, 2010-2014
}

\author{
Luis Rodolfo Améstica Rivas \\ Danilo Alexis Campos Fuentes ${ }^{* * *}$ \\ Edinson Edgardo Cornejo Saavedra ${ }^{* \text { **:* }}$
}

* doi: 10.11144/Javeriana.cao30-54.afae. Este artículo es el resultado de la investigación “Hechos esenciales y retornos en el mercado accionario en Chile", con financiamiento del Departamento de Gestión Empresarial FACE UBB. El artículo se recibió el 11/11/2016 y se aprobó 15/05/2017. Sugerencia de citación: Améstica R., L. R., Campos F., D. A., y Cornejo S., E. E. (2017). Anuncio de fusiones y adquisiciones y su efecto en los retornos accionarios: Chile, 2010-2014. Cuadernos de Administración, 30(54), 39-64. http://dx.doi. org/10.11144/Javeriana.cao30-54.afae.

** Doctor en Administración y Dirección de Empresas de la Universidad Politécnica de Cataluña, Barcelona, España, 2015. Académico Facultad de Ciencias Empresariales, Director Magíster Gestión de Empresas de la Universidad del Bío - Bío, Chile.

Correo electrónico: lamestica@ubiobio.cl

*** Magíster en Dirección de Empresas de la Universidad del Bío-Bío, Chile, 2016. Profesional de Investigación Facultad de Ciencias Empresariales de la Universidad del Bío-Bío, Chile. Correo electrónico: daacampos21@gmail.com

**** Master of Business Administration (MBA) de la Universidad Alberto Hurtado, Loyola University, 2001. Académico Facultad de Ciencia Empresariales de la Universidad del Bío-Bío, Chile.

Correo electrónico: ecornejo@ubiobio.cl 


\section{Anuncio de fusiones y adquisiciones y su efecto en los retornos accionarios: Chile, 2010-2014}

\section{RESUMEN}

Este estudio analiza 61 anuncios de fusiones y adquisiciones (F\&A) -realizados entre 2010 y 2014 por 30 firmas chilenas-y sus efectos sobre los precios accionarios; y busca retornos anormales en los días previos, que podrían relacionarse con el uso de información privilegiada y un problema de insider. Mediante un estudio de eventos se estiman los retornos anormales promedio $(A R)$ ocurridos antes y después del anuncio de F\&A. En las adquisiciones, en el día previo el $A R$ es positivo (0,60\%-0,89\%) y significativo; y el retorno anormal promedio acumulado entre los seis días previos también es positivo y significativo (2,30\%-2,48\%). Esto revelaría una filtración de información privada y un problema de insider. En las fusiones, los resultados no son concluyentes.

Palabras claves: fusión, adquisición, insider, estudio de eventos. Clasificación JEL: G14, G34

\section{Mergers and acquisitions announcements and effects on stock returns: Chile, 2010-2014}

ABSTRACT

This study analyzes 61 mergers and acquisitions (M\&A) announcements -between 2010 and 2014 by 30 Chilean firms- and their effects on share prices; and looks for abnormal returns in the previous days, which could be related to the use of privileged information and insider trading problem. Through an event study, the average abnormal returns $(A R)$ occurred before and after the announcement of $M \& A$ are estimated. In acquisitions, on the previous day the $A R$ is positive (0.60\%-0.89\%) and significant; and the cumulative average abnormal return between the previous six days is also positive and significant (2.30\%-2.48\%). This would reveal a leak of private information and insider trading problem. In mergers, the results are not conclusive.

Keywords: Merger, acquisition, insider, event studies. JEL Classification: $\mathrm{G}_{14}, \mathrm{G}_{34}$

Anúncio de fusões e aquisições e seu efeito nos retornos de ações: Chile, 2010-2014

\section{RESUMO}

Este estudo analisa 61 anúncios de fusões e aquisições (F\&A) -realizados entre 2010 e 2014 por 30 empresas chilenas-e seus efeitos sobre os preços de ações; além disso, procura retornos anormais nos dias prévios, que poderiam estar relacionados com o uso de informação privilegiada e um problema de insider. Por meio de um estudo de eventos, são estimados os retornos anormais médios (RA) ocorridos antes e depois do anúncio de F\&A. Nas aquisições, no dia prévio, o RA é positivo e significativo (0,60\%-0,89\%); o RA médio acumulado entre os seis dias prévios também é positivo e significativo (2,30\%-2,48\%). Isso revela uma possível filtração de informação privada e um problema de insider. Nas fusões, os resultados não são conclusivos.

Palavras-chave: fusão, aquisição, insider, estudo de eventos. Classificação JEL: G14, G34 


\section{Introducción}

Las fusiones y adquisiciones de firmas (F\&A) han sido una práctica empresarial común, en especial durante el siglo XX. La apertura económica y la globalización crearon la necesidad de fortalecer las empresas para ser más competitivas en costos, tamaño y tecnología, para responder más rápido a las necesidades de un mercado dinámico y cambiante.

Según Iglesias (2003), una fusión es un acto en que dos o más firmas se identifican en una sola y se puede presentar de dos formas: (1) por incorporación o absorción, donde una o más sociedades aportan todos sus activos y pasivos a una distinta, la que aumenta su capital para pagar con acciones propias el aporte realizado -hay una sociedad absorbente que adquiere la totalidad del patrimonio de otra, y una absorbida que se extingue-; y (2) por creación o fusión pura, donde dos o más sociedades se unen creando una nueva -las sociedades que se unieron desaparecen y dan origen a una nueva que adquiere todos los derechos y contrae todas las obligaciones de las firmas que se fusionaron-.

Por otra parte, una adquisición es el proceso realizado por una compañía cuando toma el control operacional de otra, mediante la compra directa o la acumulación de acciones suficientes para conseguir dicho fin, sin realizar la fusión de sus patrimonios. En una adquisición, una empresa compra los activos o acciones de otra y los accionistas de la empresa adquirida dejan de ser propietarios de esa empresa (Sudarsanam, 2003). Una adquisición puede realizarse mediante una oferta pública de adquisición de acciones $(\mathrm{OPA})$, en la que una empresa hace una oferta de compra a los accionistas de otra firma cotizada en el mercado bursátil. En este contexto, la OPA puede ser amistosa -la empresa que hace la oferta y la firma objetivo llegan a un acuerdo en relación con el precio y la forma de la operación- o puede ser hostil-la adquisición no tiene el consentimiento del equipo directivo de la empresa objetivo-.

Existen diversos motivos por los cuales las firmas realizan F\&A. Algunos autores como Santeiro (2014), Socolich (2007) y Zozaya (2007) mencionaron sinergias operacionales, sinergias financieras, aumentos de eficiencia, poder de mercado, beneficios impositivos, y uso de excedentes de fondos, entre otros motivos. Las F\&A también pueden ser una respuesta a las acciones de los competidores, donde una serie de F\&A exitosas podría conducir a que otras firmas realicen tomas de control, mientras que una serie de F\&A sin éxito podría conducir a una disminución de este tipo de operaciones. 
García (2009) señaló que, ante el anuncio o la divulgación de una noticia que contiene información relevante para la toma de decisiones, el mercado reacciona reflejando los efectos en el precio de la acción, ajustándolo al alza o a la baja, dependiendo de las expectativas futuras generadas por la nueva información disponible. Sin embargo, en ocasiones se generan variaciones en el precio de una acción antes del anuncio o publicación de una noticia relevante, lo que pudo ser consecuencia de asimetrías de información y del actuar de agentes que accedieron a la información de forma anticipada (insiders), antes de ser divulgada al público en general.

El problema es que un insider, aprovechando su posición para acceder a información relevante acerca de los planes de una firma -como una fusión, adquisición o un nuevo proyecto de inversión, entre otros-, puede anticiparse y realizar transacciones antes de que la empresa informe a las entidades reguladoras y anuncie la noticia al mercado, y obtener retornos anormalmente elevados o evitar pérdidas tras la divulgación de la información. Este comportamiento afecta valores como la lealtad en la competencia o la búsqueda de la honestidad y transparencia en las actividades mercantiles (Ried, 2004).

Este estudio analizó los anuncios de F\&A de las firmas que cotizaron sus acciones en la Bolsa de Comercio de Santiago de Chile (BCS) entre los años 2010 y 2014, y sus efectos sobre los retornos accionarios. Por medio de la metodología de estudio de eventos se midió el efecto del anuncio de una F\&A sobre el precio de la acción; y se buscó evidencia de retornos anormales previos a dicho anuncio, lo que pudo estar relacionado con el uso de información privilegiada en estos procesos y con un supuesto problema de insider.

\section{Revisión de la literatura}

\subsection{F\&A y efecto sobre el precio de la acción}

Uno de los primeros estudios sobre el efecto de las fusiones fue realizado por Mandelker (1974), quien analizó las fusiones de firmas listadas en la New York Stock Exchange (NYSE) y consumadas entre noviembre de 1941 y agosto de 1962. Mandelker (1974) concluyó que las empresas adquiridas se revaluaron en $14 \%$ como consecuencia de la fusión, mientras que las firmas compradoras no experimentaron cambios significativos. Dodd (1980) analizó las fusiones de una muestra de firmas listadas en la NYSE y mejoró el estudio de Mandelker -usó cotizaciones diarias en vez de mensuales; y estudió el efecto de la fusión en la fecha en que se anunció el inicio de las negociaciones, en vez del día en que se consumó la fusión-. Los resultados mostraron que las empresas vendedoras ganaron en 
promedio, y que las compradoras perdieron como consecuencia del anuncio de la fusión. El autor también encontró que las fusiones que fracasaron como consecuencia de una decisión del equipo directivo, ocasionaron un descenso menor en las cotizaciones que aquéllas en que las negociaciones terminaron por otras causas.

Schipper y Thompson (1983) analizaron una muestra de 55 firmas estadounidenses que anunciaron y, posteriormente, se involucraron en programas agresivos de adquisiciones. Los autores argumentaron que para las firmas compradoras, una fusión fue una fase más de un proceso de reiteradas adquisiciones. Por lo tanto, el efecto relevante de la fusión se produjo en el momento del anuncio del plan de adquisiciones. Asquith, Bruner y Mullins (1987) analizaron el efecto de la forma de pago de la fusión y encontraron que se produjeron descensos en la cotización de la firma compradora cuando las acciones se cambiaron por otras acciones, mientras que se registraron aumentos en la cotización cuando las acciones se compraron con efectivo.

Por otra parte, una de las primeras investigaciones en Estados Unidos sobre adquisiciones fue realizada por Dodd y Ruback (1977), quienes concluyeron que las firmas compradoras se revaluaron a la baja en el mes en que se produjo la oferta, en relación con el valor de 12 meses atrás; mientras que las firmas vendedoras aumentaron considerablemente su valor. Bradley (1980) realizó un estudio similar y mostró que las empresas compradoras pagaron a los accionistas de la firma objetivo un premio promedio de $49 \%$ (por las acciones que ellas compraron); y que la apreciación promedio de las acciones de la empresa objetivo, un mes después de consumada la oferta, fue de $36 \%$.

Ma, Pagán y Chu (2009) investigaron los retornos anormales de las firmas ofertantes alrededor del día del anuncio de una F\&A, para diez mercados asiáticos emergentes. Los autores encontraron que los mercados bursátiles esperaron rendimientos anormales acumulados positivos en tres ventanas de eventos diferentes: de dos días $[0 ;+1]$; de tres días $[-1 ;+1] ; y$ de cinco días $[-2 ;+2]$. Además encontraron que los efectos de la filtración de información sobre F\&A fueron estadísticamente significativos.

Yilmaz y Tanyeri (2016) examinaron una muestra global de F\&A de 47 países y encontraron que el retorno anormal promedio acumulado (CAR por sus siglas en inglés) sobre tres días fue de 6,9\% para las firmas objetivo; mientras que fue de 1,4\% para los ofertantes. Según las autoras, los resultados indicaron que la actividad de F\&A generó valor, especialmente en las transacciones donde se vendieron los derechos de control. El estudio 
también mostró que la magnitud de los CAR fue mayor en los países desarrollados que en los mercados emergentes.

Kinateder, Fabich y Wagner (2017) estudiaron los determinantes del valor de una firma, para las adquisiciones nacionales dentro de los países BRICS -Brasil, Rusia, India, China y Sudáfrica-, considerando tanto la empresa adquirente como la adquirida. En las empresas adquiridas, el retorno del anuncio fue positivo y significativo (en promedio, 1,45\%); mientras que las firmas compradoras tuvieron una pérdida leve. Además, los resultados fueron consistentes con el uso de información privilegiada.

Finalmente, Fatemi, Fooladi y Garehkoolchian (2017) evaluaron los efectos patrimoniales de corto plazo de las F\&A que tuvieron lugar en Japón, entre enero de 2000 y diciembre de 2014. Los autores encontraron que los accionistas de las empresas adquirentes no experimentaron efectos de riqueza significativos, pero los accionistas de las firmas adquiridas sí obtuvieron beneficios significativos.

\subsection{Uso de información privilegiada}

El problema de insider trading representa la figura de un individuo que se encuentra al interior de una organización, que posee o tiene acceso a información de la misma, y de la cual se sirve para realizar una transacción comercial con base en dichos datos (Velar, 2003).

De acuerdo con Leland (1992), en 1934 el Congreso de los Estados Unidos decidió que el uso de información privilegiada no era bueno para los mercados financieros, y desde entonces esta materia ha sido regulada por la Securities and Exchange Commission (SEC). La Securities Exchange Act de 1934 justificó la regulación del insider trading en la presunción de que tal actividad era injusta para los inversionistas ajenos a la organización. La línea entre qué información es justa y qué información es injusta ha sido objeto de considerables argumentos legales. Por lo anterior, hay casos estadounidenses de insider trading que enfatizaron el incumplimiento del deber fiduciario, por los empleados que usaron información privilegiada, en lugar de la injusticia (Leland, 1992). En Chile en 1994 se introdujo el Título XXI de la Ley de Mercado de Valores “De la información privilegiada" con la Ley 19.301.

Chakravarty y McConnell (1999) encontraron evidencia de una correlación positiva entre insider trading y cambios en el precio de las acciones, y revelaron que los movimientos de 
los insiders afectaron el precio de las acciones en mayor proporción que cualquier otro inversionista del mercado.

Budsaratragoona, Hillier y Lhaopadchan (2012) probaron la regulación de países desarrollados -relativa a transacciones con información privilegiada- en un entorno de mercado emergente (Tailandia), caracterizado por graves asimetrías de información, una aplicación laxa de la regulación, y baja eficiencia de los precios. La regulación tailandesa de las operaciones con información privilegiada, que imitó las reglas de un mercado desarrollado, falló en las tres medidas de éxito: los insiders transaron con impunidad durante una prohibición de transacciones, el desempeño de estos superó el de otros inversionistas, y continuaron explotando su posición privilegiada con respecto al flujo de información. Los autores sugirieron que es inapropiado que los reguladores de mercados emergentes adopten una regulación de mercado desarrollado, sin considerar las características únicas del entorno.

En otro estudio, Fidrmuc, Korczak y Korczak (2013) usaron más de 240.000 transacciones de insiders reportadas en 15 países europeos y Estados Unidos para analizar el vínculo entre la protección de los accionistas a nivel nacional y los retornos anormales de transacciones de insiders. Los autores mostraron que los retornos anormales estuvieron correlacionados positivamente con la protección de los accionistas contra la expropiación por parte de insiders corporativos. La reacción del mercado a las compras de insiders se incrementó con la protección del accionista porque esta mejoró la transparencia y la confiabilidad de las operaciones de los insiders, y limitó las posibilidades de desviar beneficios. Kraft, Soo y Lopatta (2014) encontraron que las ventas de acciones exclusivas de los ejecutivos superiores estuvieron negativamente asociadas con los retornos futuros, de lo que se infiere que dichos ejecutivos usaron información privilegiada en beneficio propio.

En otra línea de investigación, Agrawal y Cooper (2015) examinaron el uso de información privilegiada en una muestra de más de 500 empresas que estuvieron involucradas en escándalos contables, y concluyeron que el deseo de los gerentes por vender sus participaciones accionarias a precios inflados fue un motivo para la manipulación de los beneficios contables.

Balasubramnian, Fuller y Steigner (2016) examinaron el efecto de los cambios regulatorios de la SEC estadounidense en el año 2000, con respecto a la filtración de información privada antes de los anuncios de fusiones. Los autores encontraron que el volumen de transacciones anormales, debido a diferencias en la calidad de la información, se redujo 
después de la regulación -que afectó acuerdos bursátiles, firmas de alta tecnología y firmas de tamaño medio y grande-. Esto indicó una menor fuga de información después de la nueva regulación. Otro hallazgo de ese estudio fue el mayor retorno en los anuncios posteriores a la regulación, lo que mostró que los anuncios de fusiones fueron una gran sorpresa para el mercado, debido a la disminución de la filtración de información privada después de los cambios regulatorios.

En relación con el uso de información privilegiada en el mercado bursátil chileno, Vásquez (2008) analizó los periodos de publicación de los estados financieros de 42 acciones del Índice de Precios Selectivo de Acciones (IPSA), entre los años 2002 y 2007, y concluyó que existieron retornos anormales en dichos periodos. Por su parte, Ramírez y Yáñez (2010) estudiaron los movimientos de los insiders en los periodos de blackout (durante los cuales la firma prohibió a sus empleados transar acciones de la compañía), y concluyeron que estos realizaron transacciones antes y después del periodo de cierre, y obtuvieron retornos anormales.

\section{Metodología}

\subsection{Datos}

Se revisaron los anuncios de F\&A realizados entre el 1 de enero de 2010 y el 31 de diciembre de 2014 por las firmas emisoras de valores de oferta pública que estuvieron registradas en la Superintendencia de Valores y Seguros (SVS) -el organismo que supervisa y regula los mercados de valores y seguros de Chile-. Los anuncios fueron realizados por las empresas mediante la comunicación de un "hecho esencial" a la SVS. Un hecho esencial constituye un hecho relevante para la entidad fiscalizada (p. ej., un cambio en la composición del directorio, el anuncio de una fusión, etc.). Esta información es recibida y reproducida al mercado por la SVS.

Entre los años 2010 y 2014 se emitieron 11.938 hechos esenciales de diversa índole. En relación con los hechos esenciales asociados con procesos de F\&A, se encontraron tres tipos: (1) activos o paquetes accionarios, adquisición o enajenación; (2) cambios en la propiedad y/o toma de control; y (3) división, fusión o constitución de sociedades.

La muestra de estudio se definió de la siguiente manera. Primero, se identificaron las empresas que emitieron hechos esenciales sobre F\&A entre 2010 y $2014-198$ firmas con 741 hechos esenciales directa 0 indirectamente relacionados con F\&A-. Segundo, se 
identificaron los hechos esenciales donde la F\&A representó un porcentaje o participación significativa que condujo a una toma de control de la organización, es decir, donde se mencionó la toma de control operativo, reflejado en un porcentaje de participación accionaria (más del 50\%). Tercero, se seleccionaron las compañías que lograron el control operativo mediante la F\&A y, además, tuvieron una presencia bursátil mínima de $80 \%$ entre los 240 días antes de la fecha del anuncio de F\&A y los 40 días posteriores a este -para garantizar un mínimo de liquidez en las ventanas de tiempo que sugirieron Weston, Siu y Johnson (2000) en el estudio de eventos, y que el efecto de la divulgación del hecho esencial se refleje en el precio diario de la acción-.

De acuerdo con lo anterior, la muestra de estudio quedó constituida por 30 empresas, las cuales presentaron 61 anuncios de F\&A: 50 anuncios de adquisiciones (tabla 1); y 11 anuncios de fusiones (tabla 2). Las tablas 1 y 2 presentan la nómina de firmas que conformaron la muestra de estudio, el tipo de anuncio, la fecha y una descripción del acontecimiento informado.

\section{Tabla 1}

Muestra de firmas que realizaron anuncios de adquisiciones entre los años 2010 y 2014

\begin{tabular}{|c|c|c|c|c|}
\hline $\mathrm{N}^{\circ}$ & Firma & Acrónimo & Fecha & Descripción del evento \\
\hline 1 & $\begin{array}{l}\text { Viña Concha } \\
\text { y Toro }\end{array}$ & Conchatoro & $\begin{array}{l}01-03- \\
2011\end{array}$ & $\begin{array}{l}\text { Concha y Toro compró el } 100 \% \text { de las acciones de Brown-forman, } \\
\text { compañía vitivinícola norteamericana. }\end{array}$ \\
\hline 2 & $\begin{array}{l}\text { Tech Pack } \\
\text { S.A. }\end{array}$ & Techpack & $\begin{array}{l}10-06- \\
2014\end{array}$ & $\begin{array}{l}\text { La filial de la compañía Alusa S.A. adquirió el } 100 \% \text { de la } \\
\text { propiedad de la empresa chilena de productos Plásticos HYC S.A. }\end{array}$ \\
\hline 3 & Sonda S.A. & Sonda & $\begin{array}{l}23-04- \\
2010\end{array}$ & Adquirió el $100 \%$ de la propiedad de la empresa brasilera Telsinc. \\
\hline 4 & Sonda S.A. & Sonda & $\begin{array}{l}29-04- \\
2010\end{array}$ & $\begin{array}{l}\text { Adquirió el } 100 \% \text { de la propiedad de la empresa brasilera } \\
\text { Softeam. }\end{array}$ \\
\hline 5 & Sonda S.A. & Sonda & $\begin{array}{l}18-06- \\
2010\end{array}$ & $\begin{array}{l}\text { Sonda, a través de su filial Sonda México S.A., suscribió un } \\
\text { contrato de compraventa por el } 100 \% \text { de la propiedad de la } \\
\text { empresa mexicana Nextiraone México S.A. }\end{array}$ \\
\hline 6 & Sonda S.A. & Sonda & $\begin{array}{l}24-06- \\
2010\end{array}$ & $\begin{array}{l}\text { Sonda, a través de su filial en Brasil, suscribió un contrato de } \\
\text { adquisición del } 100 \% \text { de la propiedad de la empresa brasileña } \\
\text { Kaizen. }\end{array}$ \\
\hline 7 & Sonda S.A. & Sonda & $\begin{array}{l}04-11- \\
2010\end{array}$ & $\begin{array}{l}\text { Adquirió el } 100 \% \text { de la propiedad de la empresa argentina } \\
\text { Ceitech. }\end{array}$ \\
\hline 8 & Sonda S.A. & Sonda & $\begin{array}{l}10-08- \\
2011\end{array}$ & $\begin{array}{l}\text { Inició una OPA con el objetivo de adquirir el } 100 \% \text { de las acciones } \\
\text { de la sociedad Quintec S.A. }\end{array}$ \\
\hline 9 & Sonda S.A. & Sonda & $\begin{array}{l}\text { 08-03- } \\
2012\end{array}$ & Adquirió el $100 \%$ de la propiedad de la empresa brasileña Pars. \\
\hline
\end{tabular}




\begin{tabular}{|c|c|c|c|c|}
\hline $\mathrm{N}^{\circ}$ & Firma & Acrónimo & Fecha & Descripción del evento \\
\hline 10 & Sonda S.A. & Sonda & $\begin{array}{l}04-05- \\
2012\end{array}$ & $\begin{array}{l}\text { Adquirió el } 100 \% \text { de la propiedad de la sociedad Elucid Solutions } \\
\text { S.A., en Brasil. }\end{array}$ \\
\hline 11 & Sonda S.A. & Sonda & $\begin{array}{l}13-03- \\
2014\end{array}$ & $\begin{array}{l}\text { Anunció que adquirirá el } 100 \% \text { de la propiedad de la empresa } \\
\text { brasileña Ctis. }\end{array}$ \\
\hline 12 & $\begin{array}{l}\text { Sigdo } \\
\text { Koppers S.A. }\end{array}$ & Sk & $\begin{array}{l}02-08- \\
2010\end{array}$ & Adquirió el $53,73 \%$ de las acciones emitidas por Enaex. \\
\hline 13 & $\begin{array}{l}\text { Sigdo } \\
\text { Koppers S.A. }\end{array}$ & Sk & $\begin{array}{l}23-08- \\
2011\end{array}$ & $\begin{array}{l}\text { Suscribió un contrato de compraventa de acciones, en el cual SK } \\
\text { adquirirá el } 100 \% \text { de las acciones de Megotteaus Group S.A., de } \\
\text { Bélgica. }\end{array}$ \\
\hline 14 & $\begin{array}{l}\text { Sigdo } \\
\text { Koppers S.A. }\end{array}$ & Sk & $\begin{array}{l}27-10- \\
2011\end{array}$ & $\begin{array}{l}\text { La filial Ingeniería y Construcciones Sigdo S.A. adquirirá el 70\% } \\
\text { de las acciones de la Sociedad Constructora y Montajes Com S.A. }\end{array}$ \\
\hline 15 & $\begin{array}{l}\text { Salfacorp } \\
\text { S.A. }\end{array}$ & Salfacorp & $\begin{array}{l}07-01- \\
2011\end{array}$ & $\begin{array}{l}\text { Salfa Ingeniería y Construcción S.A., unidad de Salfacorp S.A., } \\
\text { adquirirá el } 100 \% \text { de la empresa Tecsa S.A. }\end{array}$ \\
\hline 16 & $\begin{array}{l}\text { Salfacorp } \\
\text { S.A. }\end{array}$ & Salfacorp & $\begin{array}{l}11-03- \\
2011\end{array}$ & $\begin{array}{l}\text { Salfa Ingeniería y Construcción S.A., unidad de Salfacorp S.A., } \\
\text { adquirirá el } 80 \% \text { de la empresa Icem S.A. }\end{array}$ \\
\hline 17 & $\begin{array}{l}\text { Salfacorp } \\
\text { S.A. }\end{array}$ & Salfacorp & $\begin{array}{l}16-05- \\
2011\end{array}$ & $\begin{array}{l}\text { Consalfa S.A., sociedad colombiana formada en partes iguales } \\
\text { por la empresa de montajes industriales Salfa S.A. y Concreto } \\
\text { S.A., acordaron la compra por parte de la primera del } 80 \% \text { de las } \\
\text { acciones de Intercoastal Marine INC (Panamá y el Caribe). }\end{array}$ \\
\hline 18 & $\begin{array}{l}\text { S.A.C.I. } \\
\text { Falabella }\end{array}$ & Falabella & $\begin{array}{l}17-09- \\
2014\end{array}$ & $\begin{array}{l}\text { La sociedad filial Sodimac Perú S.A. adquirió el } 100 \% \text { de las } \\
\text { acciones de la sociedad Maestro Perú S.A. }\end{array}$ \\
\hline 19 & $\begin{array}{l}\text { Quiñenco } \\
\text { S.A. }\end{array}$ & Quinenco & $\begin{array}{l}02-09- \\
2011\end{array}$ & $\begin{array}{l}\text { Quiñenco se comprometió a adquirir de Terpel Chile S.A. y } \\
\text { Petrolera Nacional S.A. y éstas a enajenar la totalidad de } \\
\text { sus participaciones en sus subsidiarias chilenas Petróleos } \\
\text { Trasandinos S.A. y Operaciones y Servicios Terpel Limitada. }\end{array}$ \\
\hline 20 & $\begin{array}{l}\text { Parque } \\
\text { Arauco S.A. }\end{array}$ & Parauco & $\begin{array}{l}30-03- \\
2010\end{array}$ & $\begin{array}{l}\text { Parque Arauco S.A., a través de su filial en Perú, Inmuebles } \\
\text { Comercial del Perú S.A. y Graña Montero S.A., adquiere todas las } \\
\text { acciones de G\&M en Fashion Center S.A. }\end{array}$ \\
\hline 21 & $\begin{array}{l}\text { Parque } \\
\text { Arauco S.A. }\end{array}$ & Parauco & $\begin{array}{l}21-09- \\
2010\end{array}$ & $\begin{array}{l}\text { La sociedad Inmuebles Panamericana S.A., filial de Parque } \\
\text { Arauco en Perú, celebró un contrato de compraventa en el que } \\
\text { adquirió el } 67 \% \text { del capital accionario de Altec Trading S.A.C. }\end{array}$ \\
\hline 22 & $\begin{array}{l}\text { Parque } \\
\text { Arauco S.A. }\end{array}$ & Parauco & $\begin{array}{l}20-04- \\
2012\end{array}$ & $\begin{array}{l}\text { Suscripción de un acuerdo en el que Parque Arauco S.A. adquiere } \\
\text { de Seguridad y Telecomunicaciones S.A., filial de Rebrisa S.A., el } \\
100 \% \text { de las acciones de la Sociedad Inmobiliaria Buenaventura } \\
\text { Spa. }\end{array}$ \\
\hline 23 & $\begin{array}{l}\text { Parque } \\
\text { Arauco S.A. }\end{array}$ & Parauco & $\begin{array}{l}16-10- \\
2014\end{array}$ & $\begin{array}{l}\text { La filial en Perú de Pasa Inmuebles Comerciales del Perú S.A.C. } \\
\text { adquiere el } 100 \% \text { de las acciones emitidas por Equimed S.A.C. }\end{array}$ \\
\hline 24 & $\begin{array}{l}\text { Latam } \\
\text { Airlines } \\
\text { Group S.A. }\end{array}$ & Lan & $\begin{array}{l}27-10- \\
2010\end{array}$ & $\begin{array}{l}\text { LAN celebró una promesa de compraventa por el } 98,942 \% \text { de las } \\
\text { acciones en circulación de la aerolínea colombiana Aerovías de } \\
\text { Integración Regional (Aires S.A.). }\end{array}$ \\
\hline 25 & $\begin{array}{l}\text { Inversiones la } \\
\text { Construccion } \\
\text { S.A. }\end{array}$ & Ilc & $\begin{array}{l}23-09- \\
2013\end{array}$ & $\begin{array}{l}\text { La sociedad adquirirá el } 67 \% \text { de las acciones de Corpgroup Vida } \\
\text { Chile, y la totalidad de los derechos sociales de Corpgroup Vida } \\
\text { Limitada. }\end{array}$ \\
\hline
\end{tabular}




\begin{tabular}{|c|c|c|c|c|}
\hline$N^{\circ}$ & Firma & Acrónimo & Fecha & Descripción del evento \\
\hline 26 & $\begin{array}{l}\text { Inversiones la } \\
\text { Construccion } \\
\text { S.A. }\end{array}$ & Ilc & $\begin{array}{l}19-08- \\
2014\end{array}$ & $\begin{array}{l}\text { La sociedad suscribió un acuerdo de adquisición con la sociedad } \\
\text { Baninter S.A. equivalente al 50,79\%. }\end{array}$ \\
\hline 27 & $\begin{array}{l}\text { Grupo } \\
\text { Security S.A. }\end{array}$ & Security & $\begin{array}{l}14-03- \\
2013\end{array}$ & $\begin{array}{l}\text { Adquirirá la totalidad de la participación que Sismel tiene en } \\
\text { Hipotecaria Cruz del Sur Principal S.A., que representa un 51\% } \\
\text { del capital accionario. }\end{array}$ \\
\hline 28 & $\begin{array}{l}\text { Aes Gener } \\
\text { S.A. }\end{array}$ & Aesgener & $\begin{array}{l}28-03- \\
2014\end{array}$ & Adquirirá el 50,01\% de las acciones de Guacolda. \\
\hline 29 & Besalco S.A. & Besalco & $\begin{array}{l}19-05- \\
2011\end{array}$ & $\begin{array}{l}\text { Adquisición de Kipres Ingeniero y Compañía Limitada y de } \\
\text { Servicio Aéreokipres Limitada, equivalente al } 68 \% \text { de la } \\
\text { sociedad. }\end{array}$ \\
\hline 30 & Besalco S.A. & Besalco & $\begin{array}{l}06-11- \\
2012\end{array}$ & Adquisición del 50,01\% de Construcciones Civiles S.A. \\
\hline 31 & $\begin{array}{l}\text { Bupa Chile } \\
\text { S.A. }\end{array}$ & Bupa & $\begin{array}{l}18-07- \\
2012\end{array}$ & Adquisición del $100 \%$ de la sociedad Servicio Clínico S.A. \\
\hline 32 & $\begin{array}{l}\text { Bupa Chile } \\
\text { S.A. }\end{array}$ & Bupa & $\begin{array}{l}14-09- \\
2012\end{array}$ & $\begin{array}{l}\text { Adquisición del } 100 \% \text { de la sociedad Sonorad I S.A., Sonorad II } \\
\text { S.A. y Salud Fácil S.A. }\end{array}$ \\
\hline 33 & $\begin{array}{l}\text { Bupa Chile } \\
\text { S.A. }\end{array}$ & Bupa & $\begin{array}{l}17-07- \\
2013\end{array}$ & Adquisición del 70\% de la sociedad Anglolab S.A. \\
\hline 34 & $\begin{array}{l}\text { Bupa Chile } \\
\text { S.A. }\end{array}$ & Bupa & $\begin{array}{l}10-09- \\
2013\end{array}$ & Adquisición del $100 \%$ de la sociedad Mediperu S.A. \\
\hline 35 & Cap S.A. & Cap & $\begin{array}{l}17-03- \\
2010\end{array}$ & Adquisición del 51\% de Puerto las Losas S.A. \\
\hline 36 & $\begin{array}{l}\text { Cencosud } \\
\text { S.A. }\end{array}$ & Cencosud & $\begin{array}{l}05-03- \\
2010\end{array}$ & $\begin{array}{l}\text { Acuerdo de adquisición del } 100 \% \text { de la sociedad Súper Familia } \\
\text { Comercial de Alimentos Ltda. de la ciudad de Fortaleza, Brasil. }\end{array}$ \\
\hline 37 & $\begin{array}{l}\text { Cencosud } \\
\text { S.A. }\end{array}$ & Cencosud & $\begin{array}{l}28-04- \\
2010\end{array}$ & $\begin{array}{l}\text { Acuerdo de adquisición del } 100 \% \text { de la participación de Carballo } \\
\text { Faro y Cía. Ltda. en la sociedad Perini Comercial de Alimentos } \\
\text { Ltda. de la ciudad de Salvador, Brasil. }\end{array}$ \\
\hline 38 & $\begin{array}{l}\text { Cencosud } \\
\text { S.A. }\end{array}$ & Cencosud & $\begin{array}{l}15-10- \\
2010\end{array}$ & $\begin{array}{l}\text { Acuerdo de adquisición del } 100 \% \text { de Fagparticipacoes Ltda. e } \\
\text { Irmaos Breta, Filhos e Cia Ltda., los cuales operan la cadena de } \\
\text { supermercados BRETAS. }\end{array}$ \\
\hline 39 & $\begin{array}{l}\text { Cencosud } \\
\text { S.A. }\end{array}$ & Cencosud & $\begin{array}{l}07-10- \\
2011\end{array}$ & Acuerdo de adquisición del 85,58\% de Comercial Johnson S.A. \\
\hline 40 & $\begin{array}{l}\text { Empresa } \\
\text { Nacional de } \\
\text { Electricidad } \\
\text { S.A. }\end{array}$ & Endesa & $\begin{array}{l}31-03- \\
2014\end{array}$ & $\begin{array}{l}\text { Adquisición del } 50 \% \text { de Gas Atacama Holding Limitada. Endesa } \\
\text { aceptó la oferta de venta de Southern Cross de las acciones que } \\
\text { posee en Gas Atacama. }\end{array}$ \\
\hline 41 & Enersis S.A. & Enersis & $\begin{array}{l}01-04- \\
2014\end{array}$ & Adquisición del 50\% de Gas Atacama Holding Limitada. \\
\hline 42 & $\begin{array}{l}\text { Empresa } \\
\text { IANSA S.A. }\end{array}$ & Iansa & $\begin{array}{l}07-04- \\
2010\end{array}$ & $\begin{array}{l}\text { Adquisición del } 100 \% \text { de Ed\&f Man Chile Limitada. A través de la } \\
\text { filial Iansagro S.A. }\end{array}$ \\
\hline 43 & $\begin{array}{l}\text { Empresa } \\
\text { Copec S.A. }\end{array}$ & Copec & $\begin{array}{l}14-05- \\
2010\end{array}$ & $\begin{array}{l}\text { Adquisición del } 100 \% \text { de Aeicolombia Holding Ltd. y de } \\
\text { Aeicolombia Investments Ltd., que en conjunto poseen el } 47,2 \% \\
\text { de las acciones de Proenergía International S.A. }\end{array}$ \\
\hline
\end{tabular}




\begin{tabular}{|c|c|c|c|c|}
\hline $\mathrm{N}^{\circ}$ & Firma & Acrónimo & Fecha & Descripción del evento \\
\hline 44 & $\begin{array}{l}\text { Empresa } \\
\text { Copec S.A. }\end{array}$ & Copec & $\begin{array}{l}19-05- \\
2011\end{array}$ & $\begin{array}{l}\text { Adquisición del } 51 \% \text { de Inversiones del Nordeste S.A. de } \\
\text { Colombia, a través de su filial Abastecedora de Combustible S.A. } \\
\text { (Abastible). }\end{array}$ \\
\hline 45 & $\begin{array}{l}\text { Empresa } \\
\text { Copec S.A. }\end{array}$ & Copec & $\begin{array}{l}14-03- \\
2012\end{array}$ & $\begin{array}{l}\text { Adquisición del 98,24\% de Proenergia Internacional S.A., a } \\
\text { través de la filial Copec Investments Ltd., filial de Empresas } \\
\text { Copec S,A. }\end{array}$ \\
\hline 46 & $\begin{array}{l}\text { Empresa } \\
\text { Copec S.A. }\end{array}$ & Copec & $\begin{array}{l}08-06- \\
2012\end{array}$ & $\begin{array}{l}\text { Adquisición del } 100 \% \text { de Flakeboard Company Limited., a través } \\
\text { de la filial Celulosa Arauco y Constitución S.A. y su filial Arauco } \\
\text { Canadá Ltd. }\end{array}$ \\
\hline 47 & Enjoy S.A. & Enjoy & $\begin{array}{l}25-10- \\
2012\end{array}$ & $\begin{array}{l}\text { Adquisición del } 45 \% \text { de una sociedad controladora de un hotel y } \\
\text { casino en Latinoamérica, tomando el control operativo de ésta. }\end{array}$ \\
\hline 48 & Forus S.A. & Forus & $\begin{array}{l}02-09- \\
2013\end{array}$ & Adquisición del $100 \%$ de Siete Veintes S.A. \\
\hline 49 & Forus S.A. & Forus & $\begin{array}{l}06-11- \\
2013\end{array}$ & $\begin{array}{l}\text { Adquisición del } 100 \% \text { de las acciones de la sociedad que recibirá } \\
\text { aportes de GSM Chile Limitada y de Sanmare Pty Ltd. }\end{array}$ \\
\hline 50 & Gasco S.A. & Gasco & $\begin{array}{l}29-12- \\
2010\end{array}$ & $\begin{array}{l}\text { Adquisición del } 50 \% \text { de Gazel S.A. y, en consecuencia, pasó a } \\
\text { controlar el } 100 \% \text { de la propiedad. }\end{array}$ \\
\hline
\end{tabular}

Fuente: elaboración propia, a partir de los “hechos esencial” publicados por la SVS de Chile.

\section{Tabla 2}

Muestra de firmas que realizaron anuncios de fusiones entre los años 2010 y 2014

\begin{tabular}{|c|c|c|c|c|}
\hline $\mathrm{N}^{\circ}$ & Firma & Acrónimo & Fecha & Descripción del evento \\
\hline 1 & $\begin{array}{l}\text { Viña Concha y } \\
\text { Toro }\end{array}$ & Conchatoro & $\begin{array}{l}14-07- \\
2011\end{array}$ & $\begin{array}{l}\text { Contrato entre Concha y Toro, a través de su filial VCT USA, } \\
\text { con la empresa BanfiVinstners para crear una nueva entidad } \\
\text { jurídica fusionando ambas compañías. }\end{array}$ \\
\hline 2 & $\begin{array}{l}\text { Latam Airlines } \\
\text { Group S.A. }\end{array}$ & Lan & $\begin{array}{l}13-08- \\
2010\end{array}$ & $\begin{array}{l}\text { LAN y TAM suscribieron un memorándum de entendimiento no } \\
\text { vinculante en el que expresaron la intención de combinar las } \\
\text { compañías. }\end{array}$ \\
\hline 3 & Almendral S.A. & Almendral & $\begin{array}{l}28-11- \\
2011\end{array}$ & Fusión por incorporación de GTD. \\
\hline 4 & Banmedica S.A. & Banmedica & $\begin{array}{l}30-07- \\
2010\end{array}$ & $\begin{array}{l}\text { Fusión de la filial Banmedica International SPA con la sociedad } \\
\text { Inversiones Salud Interamericana S.A. de Panamá. }\end{array}$ \\
\hline 5 & $\begin{array}{l}\text { Embotelladora } \\
\text { Andina S.A. }\end{array}$ & Andina & $\begin{array}{l}25-06- \\
2012\end{array}$ & $\begin{array}{l}\text { Fusión de Andina a través de la absorción con la Compañía } \\
\text { Embotelladora Coca-Cola Polar S.A. }\end{array}$ \\
\hline 6 & ECLS.A. & $\mathrm{Ecl}$ & $\begin{array}{l}29-01- \\
2010\end{array}$ & Fusión a través de la absorción de Inversiones Tocopilla - 1. \\
\hline 7 & Enersis S.A. & Enersis & $\begin{array}{l}29-05- \\
2013\end{array}$ & $\begin{array}{l}\text { Fusión por absorción de sus filiales Inversiones Sudamericana } \\
\text { Limitada, absorbiendo a Cono Sur. }\end{array}$ \\
\hline 8 & Enersis S.A. & Enersis & $\begin{array}{l}25-11- \\
2014\end{array}$ & $\begin{array}{l}\text { Fusión a través de la absorción de sus filiales ICT Servicios } \\
\text { Informáticos Limitada, absorbiendo a Inmobiliaria Manso de } \\
\text { Velasco Limitada. }\end{array}$ \\
\hline
\end{tabular}




\begin{tabular}{lllll}
\hline$N^{\circ}$ & \multicolumn{1}{c}{ Firma } & Acrónimo & Fecha & \multicolumn{1}{c}{ Descripción del evento } \\
\hline & $\begin{array}{l}\text { Compañía } \\
\text { General de } \\
\text { Electricidad S.A. }\end{array}$ & Cge & $\begin{array}{l}29-09- \\
2011\end{array}$ & $\begin{array}{l}\text { Se aprobó la fusión por incorporación de las filiales de CGE, de } \\
\text { Transnet S.A. con la empresa Emel S.A., la que se disolverá. }\end{array}$ \\
& $\begin{array}{l}\text { Empresa Nacio- } \\
\text { nalde Telecomu- } \\
\text { nicaciones S.A. }\end{array}$ & $28-11-$ & $\begin{array}{l}\text { Acuerdo de fusión: Entel se fusionará por incorporación con } \\
\text { GTD. }\end{array}$ \\
& $\begin{array}{l}\text { Empresa CMPC } \\
\text { S.A. }\end{array}$ & Cmpc & $\begin{array}{l}\text { 04-03- } \\
2011\end{array}$ & $\begin{array}{l}\text { Fusión de su filial Industrias Forestales S.A. con CMPC por } \\
\text { incorporación de la primera en la segunda. }\end{array}$ \\
\hline
\end{tabular}

Fuente: elaboración propia, a partir de los “hechos esencial” publicados por la SVS de Chile.

\subsection{Estimación de retornos anormales}

Se aplicó la metodología de estudio de eventos, que consiste en una estimación de los retornos anormales ocurridos antes y después de un evento en particular (Brown y Warner, 1980), evento que en esta investigación correspondió a la divulgación de un hecho esencial que anunció una F\&A. Así, se calcularon los retornos accionarios anormales de las firmas alrededor de la fecha de anuncio de una F\&A.

Primero, se identificó el día del evento $(t=0)$ como aquel en que se comunicó un hecho esencial sobre una F\&A. Luego, se definió un periodo del evento sobre la base de una ventana de 81 días: desde 40 días antes del evento $(t=-40)$-para analizar si hubo influencia de los insiders sobre el precio y el retorno de la acción en los días previos a la publicación del anuncio- hasta 40 días después del evento $(t=+40)$-para examinar cómo reaccionó el mercado a la noticia-. Se supuso que esta ventana tuvo información suficiente para describir lo que ocurrió con el retorno del activo ante el evento analizado.

Segundo, se calculó el retorno esperado para una acción $i$ en cada día $t$ del periodo del evento $\left(\hat{R}_{i t}\right)$, suponiendo condiciones normales o la ausencia del evento en estudio. Para estimar $\hat{R}_{i t}$ y detectar retornos anormales se usaron tres modelos independientes, con la finalidad de dar robustez a los resultados: (1) retorno ajustado medio; (2) modelo de mercado; y (3) retorno ajustado de mercado.

El retorno ajustado medio estima $\hat{R}_{i t}$ a partir del retorno promedio diario de un activo $\left(\bar{R}_{i t}\right)$ en un periodo sin evento, anterior a la ventana de 81 días del periodo del evento. De esta manera, $\hat{R}_{i t}$ se calculó mediante (1), donde $R_{i t}$ fue el retorno observado diario de la acción $i$ en el día $t$ de un periodo sin evento de $T=200$ días; desde $t=-241$ días hasta $t=-41$ días antes del evento. 


$$
\hat{R}_{i t}=\bar{R}_{i t}=\frac{\sum_{t=-241}^{-41} R_{i t}}{T}
$$

El modelo de mercado -fórmula (2)- estima una regresión lineal entre los retornos observados de un activo $i\left(R_{i t}\right)$ y los retornos observados de un índice bursátil representativo del mercado $\left(R_{M t}\right)$, en un periodo sin evento.

$$
R_{i t}=\alpha_{i}+\beta_{i} R_{M t}+\varepsilon_{i t}
$$

En la fórmula (2), $R_{M t}$ correspondió al retorno del Índice General de Precios de Acciones (IGPA) -de la Bolsa de Comercio de Santiago de Chile- en el día $t$ del periodo sin evento; $\beta_{i}$ midió la sensibilidad del retorno accionario de la firma $i$ a las fluctuaciones del retorno de mercado; $\alpha_{i}$ midió el retorno medio sobre el periodo, que no fue explicado por el mercado; y $\varepsilon_{i t}$ fue el error de estimación. Luego, mediante el modelo de regresión planteado por la fórmula (2) -y aplicado sobre el periodo sin evento- se obtuvo una estimación de los coeficientes $\alpha_{i}$ y $\beta_{i}\left(\hat{\alpha}_{i}\right.$ y $\hat{\beta}_{i^{\prime}}$, respectivamente). Los valores de dichos coeficientes fueron reemplazados en la fórmula (3) para estimar el retorno esperado del activo $i$ en cada día $t$ del periodo del evento $\left(\hat{R}_{i t}\right)$, a partir del retorno observado del mercado en cada día $t$ de ese periodo $\left(R_{M t}\right)$.

$$
\hat{R}_{i t}=\hat{\alpha}_{i}+\hat{\beta}_{i} R_{M t}
$$

Finalmente, el modelo del retorno ajustado de mercado es similar al anterior, pero supone que el retorno esperado de la acción $i$ es igual al retorno del mercado, en cada día $t$ del periodo del evento, tal como lo indica la fórmula (4):

$$
\hat{R}_{i t}=R_{M t}
$$

Así, el modelo del retorno ajustado de mercado supone que $\hat{\alpha}_{i}=0$ y $\hat{\beta}_{i}=1$, para cada acción. Dado que $\alpha_{i}$ usualmente es una cifra pequeña y que el promedio de los $\beta_{i}$ de todas las acciones es 1, esta aproximación produce resultados aceptables. 
Tercero, se estimó el retorno anormal o residuo de una acción $i$ para cada día $t$ del periodo del evento. El retorno anormal $\left(r_{i t}\right)$ se calculó como la diferencia entre el retorno observado de la acción $i$ en el día $t$ del periodo del evento $\left(R_{i t}\right)$ y el retorno esperado en ausencia del evento $\left(\hat{R}_{i t}\right)$, de acuerdo con la fórmula (5).

$$
r_{i t}=R_{i t}-\hat{R}_{i t}
$$

Para analizar el efecto promedio sobre los retornos accionarios de $n$ firmas, se calculó el retorno anormal promedio o residuo promedio para cada día $t$ del periodo del evento, según (6).

$$
A R_{t}=\frac{\sum_{i=1}^{n} r_{i t}}{n}
$$

Cuarto, se calculó el residuo promedio acumulado en cada día $\tau$ del periodo del evento $\left(C A R_{\tau}\right)$, de acuerdo con la fórmula (7), con $\tau=-40,-39, \ldots,+40$ días. El cálculo del $C A R_{\tau}$ se inició 40 días antes del evento (el día $t=-40$ del periodo del evento) y finalizó 40 días después de este (el día $t=+40$ del periodo del evento) -así, por ejemplo: el residuo promedio acumulado del día $\tau=-40\left(C A R_{-40}\right)$, el primer día del periodo del evento, fue igual al residuo promedio del día $t=-40\left(A R_{-40}\right)$; y el residuo promedio acumulado del día $\tau=-38\left(C A R_{-38}\right)$, el tercer día del periodo del evento, fue la suma de los residuos promedios de los días $t=-40$ a $t=\tau\left(A R_{-40}+A R_{-39}+A R_{-38}\right)-$. El CAR se usó para medir el efecto promedio acumulado del anuncio de una F\&A durante el periodo del evento, y se calculó a partir del día $t=-40$ para analizar si hubo retornos anormales acumulados antes del anuncio de una $F \& A$, como resultado de las operaciones de los insiders -en este sentido, un $C A R_{\tau}$ positivo en los días previos al anuncio de $F \& A$, entre $t=-40$ y $t=-1$, pudo indicar un uso de información privilegiada-.

$$
C A R_{\tau}=\sum_{t=-40}^{t=\tau} A R_{t}
$$

\subsection{Significancia estadística de los resultados}

Para medir la significancia estadística de los $A R_{t}$ y $C A R_{t}$ se utilizó la prueba t-Student. Se contrastaron las siguientes hipótesis nulas $\left(\mathrm{H}_{1}\right.$ y $\left.\mathrm{H}_{2}\right)$ : 
$H_{1}$ : no existieron retornos anormales promedio distintos de cero durante el periodo del evento, antes o después del anuncio de una F\&A $\left(H_{1}: A R_{t}=0\right)$.

$H_{2}$ : no existieron retornos anormales promedio acumulados distintos de cero durante el periodo del evento, antes o después del anuncio de una F\&A $\left(H_{2}: C A R_{\tau}=0\right)$.

El valor de la prueba t-Student para cada $A R_{t}\left(t_{\text {student,AR }}\right)$, con (T-1) grados de libertad, se calculó por medio de (8), donde $\hat{S}(A R)$ es la desviación estándar de los residuos promedio -calculada sobre los residuos promedio del periodo sin evento-.

$$
t_{\text {Sudent }, A R}=\frac{A R_{t}}{\hat{S}(A R)}
$$

Para medir la significancia estadística del $C A R_{t}$ se utilizó el valor de la prueba t-Student $\left(t_{\text {Student,CAR }}\right)$ según la fórmula (9), donde $\hat{S}(C A R)$ es la desviación estándar de los residuos promedio acumulados -calculada sobre los residuos promedio acumulados del periodo sin evento-. Se supuso que los retornos de las acciones se distribuyeron idéntica e independientemente.

$$
t_{\text {Studen }, C A R}=\frac{C A R_{\tau}}{\hat{S}(C A R)}=\frac{\sum_{t=-40}^{t=\tau} A R_{t}}{\sum_{t=-40}^{+40} \hat{S}(A R)}=\frac{\sum_{t=-40}^{t=\tau} A R_{t}}{\sqrt{81} \times \hat{S}(A R)}
$$

\section{Resultados}

\subsection{Adquisiciones: retorno anormal promedio}

La figura 1 muestra la evolución de los retornos anormales promedio $\left(A R_{t}\right)$ estimados por el modelo de retorno ajustado medio (modelo $A$ ), el modelo de mercado (modelo $B$ ), y el modelo de retorno ajustado de mercado (modelo C), para un periodo de 40 días antes y 40 días después del evento relativo al anuncio de una adquisición. De acuerdo con los tres modelos, se observó un $A R$ positivo alrededor del día del anuncio $(t=0)$. 


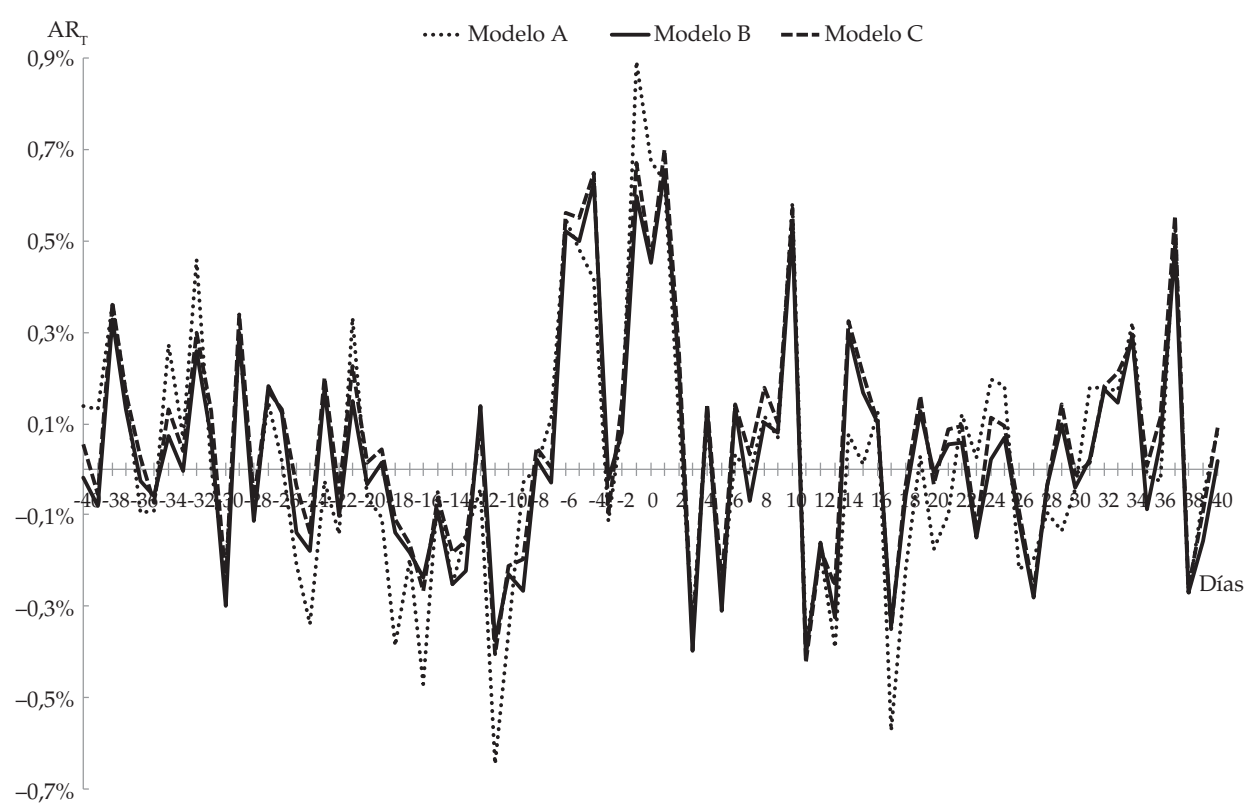

Figura 1. Retorno anormal promedio diario $\left(A R_{t}\right)$ durante el periodo del evento de anuncio de una adquisición

Fuente: elaboración propia.

La tabla 3 muestra el retorno anormal promedio $\left(A R_{t}\right)$ y el retorno anormal promedio acumulado $\left(C A R_{\tau}\right)$ de los seis días previos al anuncio de adquisición ( $t=-6$ a $\left.t=-1\right)$, del día del anuncio $(t=0)$, y de los seis días siguientes a este $(t=+1$ a $t=+6)$, junto con su significancia estadística. Se delimitó una ventana de $[-6 ;+6]$ días, dentro del periodo del evento, para focalizar el análisis en las proximidades del día del anuncio.

Se observó un $A R_{t}$ positivo y estadísticamente significativo seis días antes del anuncio de adquisición, específicamente entre los días $t=-6$ y $t=-4$, de acuerdo con los tres modelos aplicados. También se observó un $A R_{t}$ positivo un día antes del anuncio $(t=-1)$, el cual fluctuó entre 0,60\% y 0,89\%, y fue significativo con un nivel de confianza de $99 \%$, según los tres modelos. En cuanto al $C A R_{\tau^{\prime}}$, entre los días $t=-6$ y $t=-1$ fue positivo y significativo según los tres modelos, y varió entre 2,30\% y 2,48\%. Los retornos anormales promedio, positivos y significativos, de los días previos al anuncio de una adquisición, revelaron una posible fuga o filtración de información privada antes del anuncio (ver figura 2). Así, dichos retornos anormales pudieron ser el resultado de la actividad de insiders en el mercado bursátil de Chile y del uso de información privilegiada. 
En el día en que fue divulgado el hecho esencial que anunció la adquisición ( $t=0)$, el $A R_{t}$ fue positivo (entre $0,45 \%$ y $0,67 \%$ ) y estadísticamente significativo según los tres modelos. Así, se observó que los anuncios de adquisiciones tuvieron un efecto promedio positivo sobre los precios de las acciones de las firmas involucradas, lo que estuvo de acuerdo con la evidencia internacional. Luego, el $C A R_{\tau}$ entre los días $t=-6$ y $t=0$ varió entre $2,75 \%$ y $2,99 \%$, significativo con un $99 \%$ de confianza.

Además, en el día inmediatamente siguiente al anuncio ( $t=+1$ ) se observó un $A R_{t}$ entre $0,63 \%$ y $0,70 \%$, significativo de acuerdo con los tres modelos. Esto indicó que los anuncios de adquisiciones tuvieron un efecto promedio positivo sobre el precio de las acciones que se extendió hasta el siguiente día bursátil.

Los resultados también mostraron que el $C A R_{\tau}$ entre los días $t=-6$ y $t=+1$ fluctuó entre $3,40 \%$ y $3,64 \%$ y fue estadísticamente significativo. De acuerdo con esto, un insider que compró acciones en $t=-6$ ( 6 días antes del anuncio de adquisición) y las vendió en $t=+1$ (al día siguiente del anuncio), pudo obtener un retorno anormal acumulado que promedió entre $3,40 \%$ y $3,64 \%$.

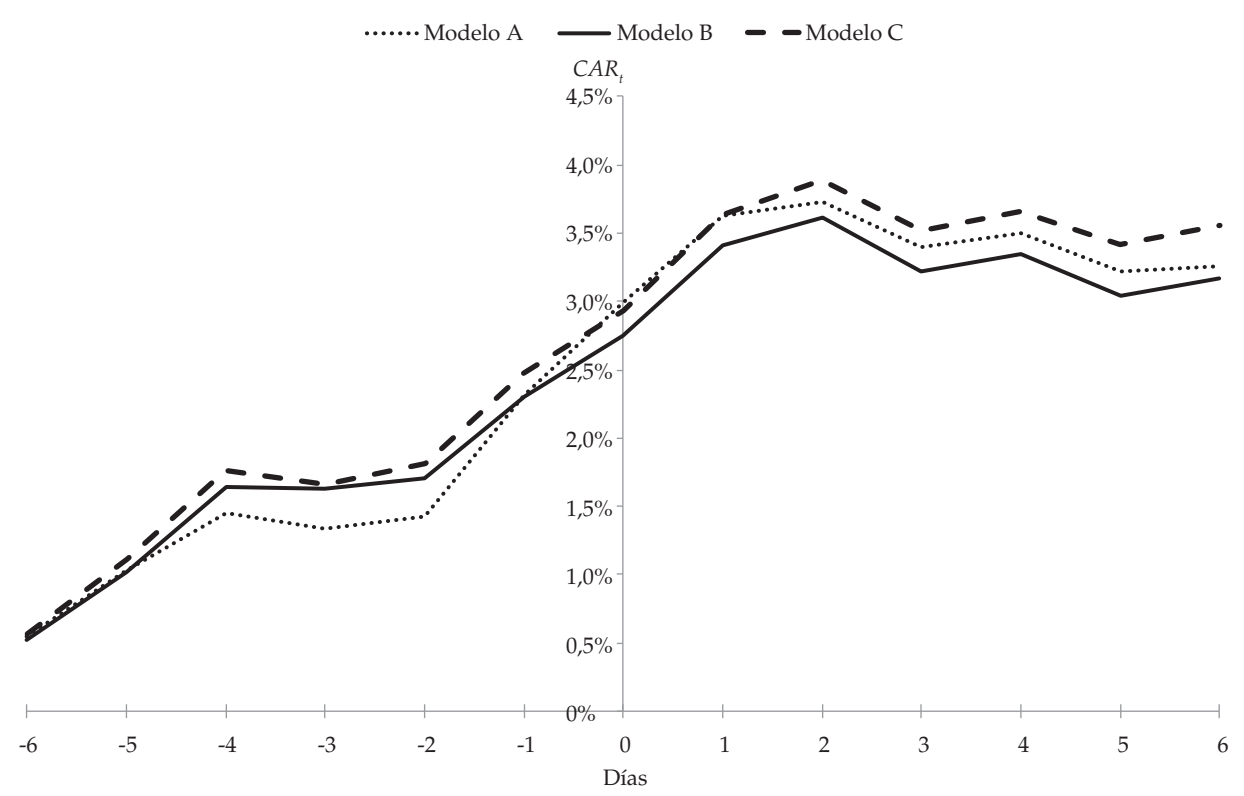

Figura 2. Retorno anormal promedio acumulado $\left(C A R_{\tau}\right)$, seis días antes y seis días después del anuncio de una adquisición

Fuente: elaboración propia. 
En los días posteriores, desde $t=+2$ y hasta $t=+40$, los $A R_{t}$ fueron tanto positivos como negativos, y en la mayoría de los casos carecieron de significancia estadística.

Sobre la base de estos resultados se rechazó la hipótesis nula de que no existieron retornos anormales distintos de cero -en promedio $\left(H_{1}\right)$ y en el acumulado $\left(H_{2}\right)$ - en los días previos al anuncio de adquisición ( $t=-6, t=-5, t=-4$ y $t=-1)$, en el día del anuncio $(t=0)$ y al día siguiente $(t=+1)$.

\section{Tabla 3}

Retornos anormales para una ventana de $[-6 ;+6]$ días en torno al anuncio de una adquisición

\begin{tabular}{|c|c|c|c|c|c|c|}
\hline \multirow{2}{*}{$\mathrm{t}$} & \multicolumn{2}{|c|}{ Modelo A } & \multicolumn{2}{|c|}{ Modelo B } & \multicolumn{2}{|c|}{ Modelo C } \\
\hline & $\mathrm{AR}_{\mathrm{t}}$ & $\mathrm{CAR}_{\tau}$ & $\mathrm{AR}_{\mathrm{t}}$ & $\mathrm{CAR}_{\tau}$ & $A R_{t}$ & $\mathrm{CAR}_{\tau}$ \\
\hline \multirow{2}{*}{-6} & $0,55 \%$ & $0,55 \%$ & $0,52 \%$ & $0,52 \%$ & $0,56 \%$ & $0,56 \%$ \\
\hline & $(2,17)^{\star \star}$ & $(2,17)^{\star \star}$ & $(2,31)^{* *}$ & $(2,31)^{\star \star}$ & $(2,40)^{\star *}$ & $(2,40)^{\star \star}$ \\
\hline \multirow{2}{*}{-5} & $0,48 \%$ & $1,03 \%$ & $0,50 \%$ & $1,02 \%$ & $0,55 \%$ & $1,11 \%$ \\
\hline & $(1,91)^{\star}$ & $(2,88)^{\star \star \star}$ & $(2,22)^{\star \star}$ & $(3,21)^{\star \star \star *}$ & $(2,35)^{\star \star}$ & $(3,36)^{\star \star \star \star}$ \\
\hline \multirow{2}{*}{-4} & $0,42 \%$ & $1,45 \%$ & $0,63 \%$ & $1,65 \%$ & $0,65 \%$ & $1,76 \%$ \\
\hline & $(1,65)^{\star}$ & $(3,30)^{\star \star \star}$ & $(2,79)^{\star \star \star}$ & $(4,23)^{* \star \star}$ & $(2,77)^{\star \star \star}$ & $(4,34)^{\star \star \star}$ \\
\hline \multirow{2}{*}{-3} & $-0,11 \%$ & $1,33 \%$ & $-0,02 \%$ & $1,62 \%$ & $-0,10 \%$ & $1,66 \%$ \\
\hline & $(-0,44)$ & $(2,64)^{\star \star \star}$ & $(-0,10)$ & $(3,61)^{\star \star \star}$ & $(-0,41)$ & $(3,55)^{\star \star \star}$ \\
\hline \multirow{2}{*}{-2} & $0,09 \%$ & $1,43 \%$ & $0,08 \%$ & $1,70 \%$ & $0,14 \%$ & $1,81 \%$ \\
\hline & $(0,37)$ & $(2,53)^{\star \star}$ & $(0,36)$ & $(3,39)^{\star \star \star}$ & $(0,62)$ & $(3,45)^{\star \star \star}$ \\
\hline \multirow{2}{*}{-1} & $0,89 \%$ & $2,32 \%$ & $0,60 \%$ & $2,30 \%$ & $0,67 \%$ & $2,48 \%$ \\
\hline & $(3,54)^{* \star \star}$ & $(3,75)^{\star \star \star}$ & $(2,66)^{\star \star \star}$ & $(4,18)^{\star * \star}$ & $(2,87)^{\star \star \star}$ & $(4,32)^{\star \star \star}$ \\
\hline \multirow{2}{*}{0} & $0,67 \%$ & $2,99 \%$ & $0,45 \%$ & $2,75 \%$ & $0,46 \%$ & $2,94 \%$ \\
\hline & $(2,67)^{\star \star \star}$ & $(4,48)^{\star \star \star}$ & $(2,00)^{\star \star}$ & $(4,63)^{\star \star \star}$ & $(1,96)^{\star \star}$ & $(4,74)^{\star \star \star \star}$ \\
\hline \multirow{2}{*}{+1} & $0,63 \%$ & $3,63 \%$ & $0,65 \%$ & $3,40 \%$ & $0,70 \%$ & $3,64 \%$ \\
\hline & $(2,50)^{* *}$ & $(5,08)^{\star * \star}$ & $(2,90)^{\star * \star}$ & $(5,35)^{\star * \star}$ & $(2,99)^{\star \star \star}$ & $(5,50)^{\star \star \star}$ \\
\hline \multirow{2}{*}{+2} & $0,11 \%$ & $3,73 \%$ & $0,21 \%$ & $3,62 \%$ & $0,25 \%$ & $3,89 \%$ \\
\hline & $(0,42)$ & $(4,93)^{\star * * *}$ & $(0,94)$ & $(5,36)^{\star \star \star}$ & $(1,08)$ & $(5,54)^{\star * \star}$ \\
\hline \multirow{2}{*}{+3} & $-0,34 \%$ & $3,40 \%$ & $-0,40 \%$ & $3,22 \%$ & $-0,37 \%$ & $3,52 \%$ \\
\hline & $(-1,33)$ & $(4,25)^{\star \star \star}$ & $(-1,78)^{\star}$ & $(4,52)^{* \star \star}$ & $(-1,57)$ & $(4,76)^{\star \star \star \star}$ \\
\hline \multirow{2}{*}{+4} & $0,10 \%$ & $3,50 \%$ & $0,14 \%$ & $3,35 \%$ & $0,14 \%$ & $3,66 \%$ \\
\hline & $(0,41)$ & $(4,18)^{\star \star \star}$ & $(0,60)$ & $(4,49)^{\star \star \star}$ & $(0,60)$ & $(4,72)^{\star \star \star}$ \\
\hline
\end{tabular}




\begin{tabular}{ccccccc}
\hline \multirow{2}{*}{$\mathrm{t}$} & \multicolumn{2}{c}{ Modelo A } & \multicolumn{2}{c}{ Modelo B } & \multicolumn{2}{c}{ Modelo C } \\
\cline { 2 - 7 } & $\mathrm{AR}_{\mathrm{t}}$ & $\mathrm{CAR}_{\tau}$ & $\mathrm{AR}_{\mathrm{t}}$ & $\mathrm{CAR}_{\tau}$ & $\mathrm{AR}_{\mathrm{t}}$ & CAR $_{\tau}$ \\
\hline \multirow{2}{*}{+5} & $-0,28 \%$ & $3,22 \%$ & $-0,31 \%$ & $3,04 \%$ & $-0,25 \%$ & $3,41 \%$ \\
& $(-1,10)$ & $(3,69)^{* * *}$ & $(-1,39)$ & $(3,90)^{* \star *}$ & $(-1,07)$ & $(4,21)^{* * *}$ \\
& $0,03 \%$ & $3,26 \%$ & $0,13 \%$ & $3,17 \%$ & $1,14 \%$ & $3,55 \%$ \\
+6 & $(0,13)$ & $(3,58)^{* * *}$ & $(0,60)$ & $(3,91)^{\star * *}$ & $(0,60)$ & $(4,21)^{* * *}$ \\
\hline
\end{tabular}

Nota: el día $t=0$ fue el día en que se anunció una adquisición, por parte de las firmas consideradas en el estudio. Se presentan los resultados para una ventana de $[-6 ;+6]$ días en torno al anuncio, no obstante el análisis se realizó para una ventana (período del evento $)$ de $[-40 ;+40]$ días. El retorno esperado $\left(\hat{R}_{i+}\right)$ y, por lo tanto, el retorno anormal $\left(r_{i t}\right)$, el retorno anormal promedio $\left(A R_{t}\right)$ y el retorno anormal promedio acumulado $\left(C A R_{\tau}\right)$, fueron calculados mediante tres modelos independientes -retorno ajustado medio (A), modelo de mercado (B) y retorno ajustado de mercado (C)-, para dar robustez a los resultados. Entre paréntesis se muestra el valor de la prueba t-Student para el $A R_{t}$ y el $C A R_{\tau}$ de cada modelo (con $\left.\tau=-6,-5, \ldots,+6\right)$, con significancia del *: $90 \%,{ }^{* *}: 95 \%, y^{* * *}: 99 \%$ de confianza.

Fuente: elaboración propia.

\subsection{Fusiones: retorno anormal promedio}

La figura 3 muestra la evolución de los retornos anormales promedio $\left(A R_{t}\right)$ estimados por el modelo de retorno ajustado medio (modelo A), el modelo de mercado (modelo B), y el modelo de retorno ajustado de mercado (modelo C), para un periodo de 40 días antes y 40 días después del anuncio de una fusión. Se observó, según los tres modelos, un $A R_{t}$ positivo en el día del evento $(t=0)$.

La tabla 4 muestra los resultados más relevantes: los $A R_{t}$ y $C A R_{\tau}$ de los cuatro días previos al anuncio ( $t=-4$ a $t=-1)$, del día del anuncio $(t=0)$, y de los cuatro días posteriores a este $(t=+1$ a $t=+4)$. Se delimitó una ventana de $[-4 ;+4]$ días, dentro del periodo del evento, para focalizar el análisis en las cercanías del día del anuncio.

Se observó un $A R_{t}$ positivo y estadísticamente significativo cuatro días antes del anuncio de una fusión (en $t=-4$ ), de acuerdo con los tres modelos. Dos día antes del anuncio (en $t=-2)$, el $A R_{t}$ fue positivo, pero no fue significativo. Y se observó un $A R_{t}$ negativo un día antes del anuncio (en $t=-1$ ), entre $-0,78 \%$ y $-0,47 \%$, el cual fue significativo según el modelo del retorno ajustado medio y el modelo de mercado. El $C A R_{\tau}$ entre los días $t=-4$ y $t=-1$ varió entre $-0,20 \%$ y $0,87 \%$ y no fue significativo. Así, los resultados no fueron concluyentes con respecto a la actividad de insider y al uso de información privilegiada en los días previos al anuncio de una fusión, ya que los $A R_{t}$ fueron mixtos (positivos y negativos) y no siempre significativos. 


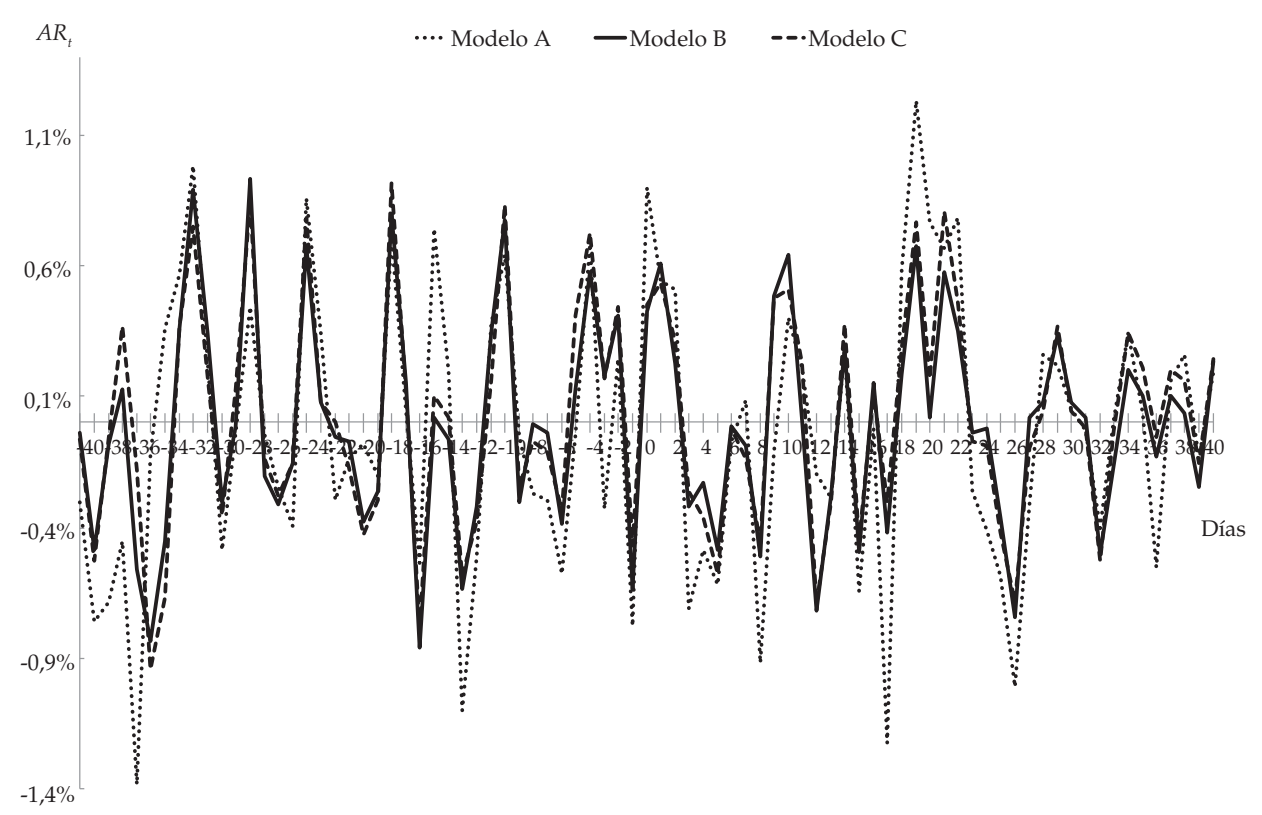

Figura 3. Retorno anormal promedio diario $\left(A R_{t}\right)$ durante el periodo del evento de anuncio de una fusión

Fuente: elaboración propia.

En el día en que fue divulgado el hecho esencial que anunció la fusión ( $t=0)$, el $A R_{t}$ fue positivo, entre $0,43 \%$ y $0,90 \%$, pero fue significativo sólo según las estimaciones del modelo de retorno ajustado medio. Así, se observó que los anuncios de fusiones tuvieron un efecto promedio positivo sobre los precios de las acciones de las firmas involucradas, pero la significancia de los resultados no fue tan robusta como en el caso de las adquisiciones. Luego, el $C A R_{\tau}$ entre los días $t=-6$ y $t=0$ fue entre $0,72 \%$ y $1,32 \%$, significativo sólo según el modelo de retorno ajustado de mercado.

Además, al día siguiente del anuncio $(t=+1)$ se observó un $A R_{t}$ positivo, entre $0,54 \%$ y $0,61 \%$, significativo de acuerdo con el modelo de mercado. Esto indicó que los anuncios de fusiones tuvieron un efecto promedio positivo sobre el precio de las acciones que se extendió hasta el siguiente día bursátil, no obstante, la significancia estadística de este resultado no fue tan robusta.

Se encontró que en el día $t=+2$ el $A R_{t}$ fue positivo, pero no significativo. Sin embargo, el $C A R_{\tau}$ entre los días $t=-4$ y $t=+2$ fue positivo y significativo, según los tres modelos, lo 
que evidenció un efecto acumulado positivo sobre el precio de las acciones. Más allá de $t=+2$ el $C A R_{\tau}$ perdió significancia estadística; y en $t=+3$ y $t=+4$ el $A R_{t}$ fue negativo, pero no significativo.

\section{Tabla 4}

Retornos anormales para una ventana de [-4;+4] días en torno al anuncio de una fusión

\begin{tabular}{|c|c|c|c|c|c|c|}
\hline \multirow{2}{*}{$\mathrm{t}$} & \multicolumn{2}{|c|}{ Modelo A } & \multicolumn{2}{|c|}{ Modelo B } & \multicolumn{2}{|c|}{ Modelo C } \\
\hline & $\mathrm{AR}_{\mathrm{t}}$ & $\mathrm{CAR}_{\tau}$ & $\mathrm{AR}_{\mathrm{t}}$ & $\mathrm{CAR}_{\tau}$ & $\mathrm{AR}_{\mathrm{t}}$ & $\mathrm{CAR}_{\tau}$ \\
\hline \multirow{2}{*}{-4} & $0,69 \%$ & $0,69 \%$ & $0,57 \%$ & $0,57 \%$ & $0,73 \%$ & $0,73 \%$ \\
\hline & $(1,73)^{\star}$ & $(1,73)^{*}$ & $(1,77)^{*}$ & $(1,77)^{\star}$ & $(2,10)^{\star \star}$ & $(2,10)^{* *}$ \\
\hline \multirow{2}{*}{-3} & $-0,33 \%$ & $0,36 \%$ & $0,18 \%$ & $0,75 \%$ & $0,17 \%$ & $0,89 \%$ \\
\hline & $(-0,84)$ & $(0,63)$ & $(0,54)$ & $(1,63)$ & $(0,49)$ & $(1,83)^{\star}$ \\
\hline \multirow{2}{*}{-2} & $0,24 \%$ & $0,60 \%$ & $0,41 \%$ & $1,16 \%$ & $0,45 \%$ & $1,34 \%$ \\
\hline & $(0,61)$ & $(0,87)$ & $(1,25)$ & $(2,06)^{\star *}$ & $(1,29)$ & $(2,24)^{\star *}$ \\
\hline \multirow{2}{*}{-1} & $-0,78 \%$ & $-0,20 \%$ & $-0,63 \%$ & $0,52 \%$ & $-0,47 \%$ & $0,87 \%$ \\
\hline & $(-1,96)^{\star \star}$ & $(-0,23)$ & $(-1,96)^{\star \star}$ & $(0,80)$ & $(-1,36)$ & $(1,26)$ \\
\hline \multirow{2}{*}{0} & $0,90 \%$ & $0,72 \%$ & $0,43 \%$ & $0,95 \%$ & $0,45 \%$ & $1,32 \%$ \\
\hline & $(2,28)^{\star *}$ & $(0,82)$ & $(1,31)$ & $(1,31)$ & $(1,31)$ & $(1,71)^{\star}$ \\
\hline \multirow{2}{*}{+1} & $0,54 \%$ & $1,27 \%$ & $0,61 \%$ & $1,56 \%$ & $0,54 \%$ & $1,86 \%$ \\
\hline & $(1,37)$ & $(1,31)$ & $(1,89)^{\star}$ & $(1,96)^{\star \star}$ & $(1,55)$ & $(2,20)^{\star \star}$ \\
\hline \multirow{2}{*}{+2} & $0,51 \%$ & $1,78 \%$ & $0,23 \%$ & $1,78 \%$ & $0,31 \%$ & $2,17 \%$ \\
\hline & $(1,29)$ & $(1,7)^{*}$ & $(0,70)$ & $(2,08)^{* *}$ & $(0,89)$ & $(2,37)^{\star \star}$ \\
\hline \multirow{2}{*}{+3} & $-0,71 \%$ & $1,07 \%$ & $-0,32 \%$ & $1,47 \%$ & $-0,26 \%$ & $1,91 \%$ \\
\hline & $(-1,79)^{\star}$ & $(0,95)$ & $(-0,98)$ & $(1,60)$ & $(-0,75)$ & $(1,95)^{\star}$ \\
\hline \multirow{2}{*}{+4} & $-0,49 \%$ & $0,58 \%$ & $-0,23 \%$ & $1,24 \%$ & $-0,36 \%$ & $1,54 \%$ \\
\hline & $(-1,23)$ & $(0,49)$ & $(-0,71)$ & $(1,27)$ & $(-1,05)$ & $(1,49)$ \\
\hline
\end{tabular}

Nota: el día $t=0$ fue el día en que se anunció una fusión, por parte de las firmas de la muestra de estudio. Los resultados se presentan para una ventana de $(-4 ;+4)$ días en torno al evento (no obstante, el análisis se realizó para una ventana de $[-40$; +40] días). El retorno esperado $\left(\hat{R}_{i t}\right) \mathrm{y}$, por lo tanto, el retorno anormal $\left(r_{i t}\right)$, el retorno anormal promedio $\left(A R_{t}\right)$ y el retorno anormal promedio acumulado $\left(C A R_{\tau}\right)$, fueron calculados mediante tres modelos independientes -retorno ajustado medio $(A)$, modelo de mercado (B) y retorno ajustado de mercado (C)-, para dar robustez a los resultados. Entre paréntesis se muestra el valor de la prueba t-Student para el $A R_{t}$ y el $C A R_{\tau}$ de cada modelo (con $\left.\tau=-4,-3, \ldots,+4\right)$ con significancia del *: $90 \%,{ }^{* *}: 95 \%$, $y^{* * *}: 99 \%$ de confianza.

Fuente: elaboración propia.

Sobre la base de estos resultados se rechazó la hipótesis nula de que no existieron retornos anormales distintos de cero -en promedio $\left(H_{1}\right)$ y en el acumulado $\left(H_{2}\right)$ - cuatro días antes del anuncio de una fusión. Y también se rechazó $H_{1}$ para el día previo al anuncio, donde el retorno anormal promedio fue negativo y significativo. No obstante, no se en- 
contró suficiente evidencia para rechazar la hipótesis nula en el día del anuncio $(t=0)$ y al día siguiente de este $(t=+1)$.

\section{Conclusiones de la investigación}

Esta investigación, mediante un estudio de eventos, midió el efecto del anuncio de una F\&A sobre el precio de la acción; y buscó evidencia de retornos anormales previos a dicho anuncio, lo que pudo estar relacionado con un supuesto problema de insider. Para ello se usaron los anuncios de F\&A de las firmas que cotizaron sus acciones en la Bolsa de Comercio de Santiago de Chile (BCS) entre los años 2010 y 2014. Los resultados mostraron que los anuncios generaron efectos distintos sobre los retornos accionarios, dependiendo de si fueron acerca de adquisiciones o fusiones.

En relación con las adquisiciones, se observó un $A R_{t}$ positivo y estadísticamente significativo seis días antes del anuncio, específicamente entre los días $t=-6$ y $t=-4$. También se observó un $A R_{t}$ positivo y significativo un día antes del anuncio $(t=-1)$. Y el $C A R_{t}$ entre los días $t=-6$ y $t=-1$ fue positivo, estadísticamente significativo, y varió entre 2,30\% y 2,48\%.

En el día en que se anunció la adquisición, el $A R_{t}$ fue positivo y significativo (entre $0,45 \%$ y $0,67 \%$ ). Así, se observó que los anuncios de adquisiciones tuvieron un efecto promedio positivo y significativo sobre los precios de las acciones de las firmas involucradas, efecto que se extendió hasta el siguiente día bursátil.

Los resultados mostraron retornos anormales (promedios y acumulados) positivos y significativos en los días previos al anuncio de una adquisición, y revelaron una posible fuga o filtración de información privada antes de este. Esto coincidió con los resultados de otros estudios -como Ma et al. (2009) y Kinateder et al. (2017)- que también encontraron evidencia de filtración de información sobre F\&A y uso de información privilegiada. Así, estos retornos anormales pudieron ser el resultado de las operaciones de insiders. De acuerdo con esto, un insider que compró acciones seis días antes del anuncio de adquisición y luego las vendió al día siguiente del anuncio, pudo obtener un retorno anormal acumulado que promedió entre $3,40 \%$ y $3,64 \%$.

En el caso de las fusiones, los resultados no fueron concluyentes con respecto al comportamiento del retorno anormal promedio en los días previos al anuncio. Los retornos anormales promedio, previos al anuncio de una fusión, fueron mixtos (positivos y negativos) y no siempre significativos, por lo que no se identificó un patrón discernible 
en el comportamiento de estos. En el día del anuncio de la fusión, se observó un efecto promedio positivo sobre los precios de las acciones de las firmas involucradas, pero la significancia de los resultados no fue tan robusta como en el caso de las adquisiciones. Además, los anuncios de fusiones tuvieron un efecto promedio positivo que se extendió hasta el siguiente día bursátil, no obstante, la significancia estadística de este resultado no fue tan robusta. El tamaño relativamente pequeño de la muestra (once anuncios de fusiones) pudo afectar negativamente la magnitud y significancia de los resultados.

En relación con las limitaciones del estudio, la medición de la significancia estadística de los resultados $\left(A R_{t}\right.$ y $\left.C A R_{\tau}\right)$ se basó en una prueba paramétrica (t-Student). Al respecto, Corrado (1989) y Cowan (1992) señalaron que una desventaja de la estadística paramétrica es que incorpora supuestos detallados acerca de la distribución de probabilidad de los retornos. En este caso, el supuesto de una distribución normal de los retornos pudo reducir la capacidad de la prueba t-Student para detectar retornos anormales alrededor del anuncio de una F\&A. Por lo anterior, una futura investigación debería utilizar pruebas no paramétricas para continuar y profundizar el estudio de este tema. No obstante, los resultados del estudio, especialmente aquellos relacionados con los anuncios de adquisiciones, tendrían consecuencias sobre los futuros cambios en la regulación del mercado bursátil de Chile, ya que implicarían un refinamiento de las reglas de uso de información privilegiada, y una revisión de la regulación de F\&A.

\section{Referencias}

Agrawal, A., and Cooper, T. (2015). Insider trading before accounting scandals. Journal of Corporate Finance, 34, 169-190.

Asquith, P., R. Bruner, and D. Mullins, Jr. (1987). Merger Returns and the Form of Financing. Proceedings of the Seminar on the Analysis of Security Prices, 34, 115-146.

Balasubramnian, B., Fuller, K., and Steigner, T. (2016). Changes in information environment and merger announcements. American Journal of Business, 31(3), 123-145.

Bradley, M. (1980). Interfirm Tender Offers and the Market for Corporate Control. The Journal of Business, 53(4), 345-376.

Brown, S., and Warner, J. (1980). Measuring security price performance. Journal of Financial Economics, 8, 205-258.

Budsaratragoona, P., Hillier, D., and Lhaopadchan, S. (2012). Applying developed-country regulation in emerging markets: an analysis of Thai insider trading. Accounting and Finance, 52, 1013-1039. 
Castilla, J. A. (2011). Impacto de las fusiones y adquisiciones en la banca colombiana, 1996-2011. Bogotá: Pontificia Universidad Javeriana.

Chakravarty, S., and McConnell, J. (1999). Does Insider Trading Really Move Stock Prices? Journal of Financial and Quantitative Analysis, 34(2), 191-209.

Corrado, C. (1989). A nonparametric test for abnormal security-price performance in event studies. Journal of Financial Economics, 23(2), 385-395.

Cowan, A. R. (1992). Nonparametric event study tests. Review of Quantitative Finance and Accounting, 2(4), 343-358.

Dodd, P. (1980). Merger proposals, management discretion and stockholder wealth. Journal of Financial Economics, 8(2), 105-135.

Dodd, P., and Ruback, R. (1977). Tender Offers and Stockholder Returns: An Empirical Analysis. Journal of Financial Economics, 5(3), 351-373.

Fatemi, A. M., Fooladi, I., and Garehkoolchian, N. (2017). Gains from mergers and acquisitions in Japan. Global Finance Journal, 32, 166-178.

Fidrmuc, J., Korczak, A., and Korczak, P. (2013). Why does shareholder protection matter for abnormal returns after reported insider purchases and sales? Journal of Banking and Finance, 37(6), 1915-1935.

García, J. J. (2009). Los estudios de acontecimientos y la importancia de la metodología de estimación. Lecturas de Economía, 70, 223-235.

Iglesias, C. (2003). Transformación, división y fusión de sociedades anónimas (Licenciatura en Ciencias Juridicas y Sociales). Santiago: Universidad de Chile.

Kinateder, H., Fabich, M., and Wagner, N. (2017). Domestic mergers and acquisitions in BRICS countries: Acquirers and targets. Emerging Markets Review, 32, 190-199.

Kraft, A., Soo, B., and Lopatta, K. (2014). Management earnings forecasts, insider trading, and information asymmetry. Journal of Corporate Finance, 26, 96-123.

Leland, H. E. (1992). Insider Trading: Should It Be Prohibited? Journal of Political Economy, 100(4), 859-887.

Ma, J., Pagán, J. A., and Chu, Y. (2009). Abnormal returns to mergers and acquisitions in ten asian stock markets. International Journal of Business, 14(3), 235-250.

Mateev, M. (2017). Is the M\&A announcement effect different across Europe? More evidences from continental Europe and the UK. Research in International Business and Finance, 40, 190-216.

Mandelker, G. (1974). Risk and return: The case of merging firms. Journal of Financial Economics, 1(4), 303-335.

Persons, J., and Warther, V. (1997). Boom and bust patterns in the adoption of financial innovations. Review of Financial Studies, 10(4), 939-967. 
Ramírez, V., and Yáñez, G. (2010). Información en los estados financieros y periodos de Blackout: Evidencia para Chile. Santiago: Superintendencia de Valores y Seguros.

Ried, J. (2004). Fundamentos de la prohibición del uso de información privilegiada en Chile: una visión crítica. Revista Chilena de Derecho, 31, 439-463.

Santeiro, G. (2014). Cuestiones básicas y metodología en el proceso de fusiones y adquisiciones. Tesis de maestría. Universidad de Coruña, España.

Schipper, K., and Thompson, R. (1983). Evidence on the Capitalized Value of Merger Activity for Acquiring Firms. Journal of Financial Economics, 11(1-4), 85-119.

Socolich, M. (2007). Desempeño financiero y creacion de valor en empresas chilenas fusionadas. Tesis. Santiago: Universidad de Chile.

Sternberg, E. (2000). Just business: business ethics in action. 0xford: Oxford University Press.

Sudarsanam, P. S. (1995). The essence of mergers and acquisitions. Hertfordshire: Prentice Hall International.

Sudarsanam, S. (2003). Creating value from mergers and acquisitions: The challenges. Malaysia: Pearson Education Limited.

Vásquez, E. (2008). Retornos accionarios anormales alrededor de la fecha de publicación de los estados financieros. Santiago: Superintendencia de Valores y Seguros.

Velar, J. C. (2003). La informacion privilegiada en el mercado de capitales (Ley 18.045). Monografía. Santiago: Universidad de Chile.

Weston, J. F., Siu, J. A., and Johnson, B. A. (2000). Takeovers, restructuring, \& corporate governance. NJ: Prentice Hall.

Yılmaz, I. S., and Tanyeri, B. (2016). Global merger and acquisition (M\&A) activity, 1992-2011. Finance Research Letters, 17, 110-117.

Zozaya, N. Z. (2007). Las fusiones y adquisiciones como fórmula de crecimiento empresarial. Dirección General de Política de la PYME. Madrid, España. 